Revue européenne des sciences sociales

European Journal of Social Sciences

XLIV-134 | 2006

Quel(s) défi(s) pour les sciences sociales à l'heure de la mondialisation?

\title{
Stabilité et fluctuations de la foi chrétienne. La foi et ses expressions dans une perspective théologique
}

Pierre-Luigi Dubied

\section{CpenEdition}

\section{Journals}

Édition électronique

URL : http://journals.openedition.org/ress/289

DOI : $10.4000 /$ ress.289

ISSN : 1663-4446

Éditeur

Librairie Droz

Édition imprimée

Date de publication : 1 juin 2006

Pagination : 119-128

ISBN : 9-782-600-01095-5

ISSN : 0048-8046

\section{Référence électronique}

Pierre-Luigi Dubied, «Stabilité et fluctuations de la foi chrétienne. La foi et ses expressions dans une perspective théologique », Revue européenne des sciences sociales [En ligne], XLIV-134 | 2006, mis en ligne le 14 octobre 2009, consulté le 10 décembre 2020. URL : http://journals.openedition.org/ress/ 289 ; DOI : https://doi.org/10.4000/ress.289 
Pierre-Luigi DUBIED

\section{STABILITÉ ET FLUCTUATIONS DE LA FOI CHRÉTIENNE. LA FOI ET SES EXPRESSIONS DANS UNE PERSPECTIVE THÉOLOGIQUE}

Peut-être le public se fait-il une représentation monolithique de la foi chrétienne? De même arrive-t-il parfois à des croyants d'être hantés de scrupules en s'imaginant qu'à la perfection, ils devraient vivre d'une foi solide, arrêtée, compacte et fixe. Certains incroyants, rendus attentifs à un type de discours religieux peuvent, sans malice, supposer que la foi consiste à adopter d'un coup un ensemble de croyances sans fondements empiriques. Ils se font alors la représentation d'une foi essentiellement fanatique.

La foi tombe-t-elle du ciel? Et si quelque chose devait être considéré comme émanant du ciel, quoi, au juste?

Nous nous efforcerons de dégager ici quelques traits susceptibles de montrer comment la foi s'est façonnée et se constitue. Nous aurons donc la prétention de vérifier la représentation qu'on s'en fait ainsi que la solidité du thème de la stabilité et des fluctuations de la foi religieuse, en l'occurrence chrétienne. Du même coup nous en proposerons une définition. Nous allons suivre l'inscription de la foi dans le temps, déceler son rapport au temps entre stabilité et fluctuation.

\section{PRÉAMBULE}

Pour aborder notre problématique, il convient peut-être de commencer par rappeler que la foi chrétienne commence avec l'événement de Pâques. C'est à Pâques, à travers la confession de Jésus mort et ressuscité, que s'exprime pour la première fois ce qu'on doit appeler la foi chrétienne: la foi attachée au Christ, c'est-à-dire au Messie.

Et déjà l'usage de ce dernier terme «Messie» doit nous alerter. Il est la transposition française (ou allemande «Messias», ou italienne «Messia» ou anglaise «Messiah», etc.) d'un mot hébreu («Meshiach»), de même que le titre «Christ» est la transposition du mot grec qui traduit précisément l'hébreu. La foi au «Messie» manifeste, par conséquent, l'origine et les antécédents de la foi chrétienne dans la tradition juive. Cela devient encore plus évident lorsqu'on réalise que l'homme Jésus de Nazareth, à qui s'attache le titre de Christ, était lui-même un croyant juif qui partageait la foi religieuse de son peuple.

On doit donc commencer par reconnaître que la foi chrétienne a une importante préhistoire et que, de ce point de vue déjà, elle se situe entre stabilité et fluctuation. Certes, la foi chrétienne innove en rattachant la figure messianique à Jésus de Nazareth, et cette innovation va très tôt provoquer sa séparation du Judaïsme. Simultanément, la foi chrétienne reprend la foi d'Israël et la foi juive, comme en 
témoignent par exemple les très nombreuses références à l'Ancien Testament $(=\mathrm{AT})$ et citations de l'AT dans le Nouveau Testament $(=\mathrm{NT})$, c'est-à-dire le rattachement explicite des auteurs du NT à la tradition d'Israël.

Il n'est donc nullement exagéré de prétendre que la foi chrétienne trouve sa préhistoire dans la foi exprimée à diverses époques par des représentants du peuple d'Israël.

Or la foi d'Israël a elle-même une histoire, complexe, entre stabilité et fluctuation. Pour exemple, prenons le thème du monothéisme: tous les spécialistes de l'AT sont d'accord pour dire qu'il s'est peu à peu dégagé d'un ensemble de vénérations des dieux des ancêtres reconnus par le peuple, et qu'il n'a pas été posé au début de l'histoire comme un décret. Autre exemple de stabilité et de fluctuation inscrit dans le parcours du temps: à ses débuts, le monothéisme israélite a été essentiellement rattaché à la souveraineté nationale; Dieu était conçu comme le Dieu des douze tribus, qui les avait conduites à l'indépendance et à la sédentarisation, accomplissant ainsi la promesse séculaire rattachée à Abraham. Mais en 587 avant J.-C., Israël perdit son indépendance et la foi en Dieu entra dans une crise dont témoignent nombre d'écrits de l'AT. La foi si stable sur ce point pendant quelques siècles avait perdu une de ses assises par l'invasion babylonienne, la déportation des élites et la mise à sac du pays: Israël avait perdu son autonomie, toute souveraineté. Dès lors la foi en Dieu dut se rétablir par rapport aux événements et restaurer le thème de la promesse. Le prophète Esaïe alla, au grand scandale d'une partie de son public, jusqu'à vouloir reconnaître dans le roi païen perse Cyrus, qui mit fin à l'hégémonie de Babylone, un instrument de la volonté de Dieu (Esaïe 41, 2-3 + 25; 44, 28-45, 1).

La foi dont est issue la foi chrétienne en ligne directe était une foi historique, liée à un Dieu reconnu d'abord comme le souverain de l'histoire. Cela la distinguait de la plupart des cultes entretenus dans les cultures qui jouxtaient Israël, qui étaient plutôt attachés à la nature et à la célébration des forces qui s'y manifestaient.

C'est ainsi qu'à travers le Christianisme, l'ancien Israël et le Judaïsme apportèrent à la culture occidentale l'essentiel de sa conscience historique.

D'entrée nous percevons donc que le problème de la stabilité de la foi et de ses fluctuations se présentera de façon plus complexe qu'on aurait pu le prévoir.

\section{QUELQUES POINTS D'HISTOIRE DE LA FOI CHRÉTIENNE}

Et cela dès les débuts.

La foi chrétienne est née à Pâques, dans la proclamation du crucifié ressuscité: nous avons là le noyau stable de toute foi chrétienne, exprimé très tôt dans les premières et élémentaires confessions de foi. L'apôtre Paul rapporte ainsi quelques formules anciennes qui condensent la foi des premiers chrétiens : «Je vous ai transmis en premier lieu ce que j'avais reçu moi-même: Christ est mort pour nos péchés, selon les Ecritures. Il a été enseveli, il est ressuscité le troisième jour, selon les Ecritures. Il est apparu à Céphas, puis aux Douze. Ensuite il est apparu à plus de cinq cents frères à la fois; la plupart sont encore vivants et 
quelques-uns sont morts.» [Corinthiens 15, 3-5] ou, autre exemple: «(...) nous croyons en Celui qui a ressuscité d'entre les morts Jésus notre Seigneur, livré pour nos fautes et ressuscité pour notre justification.» [Romains 4, 24s]

Aussitôt, je me trouve contraint de faire part d'un résultat étrange de la recherche en sociologie du Christianisme primitif. Il se révèle aujourd'hui hautement probable qu'à côté et en plus des plus anciennes communautés chrétiennes qui vivaient leur foi autour de la confession que je viens d'évoquer, il faut compter avec un courant important de prophètes missionnaires directement rattachés au souvenir de Jésus, qui n'accordaient aucune importance théologique à son destin et à sa mort: ces prédicateurs itinérants radicaux avaient, avant tout, retenu certaines paroles de Jésus, le mode de vie qu'il avait illustré, et se reconnaissaient chargés de perpétuer son message dans la hâte. Pour eux, en effet, l'histoire allait tantôt prendre fin. Faut-il juger que ces gens étaient en dehors de la foi chrétienne? Oui, bien sûr. Et cependant, ils étaient mus par une foi en Dieu dont le truchement était la personne de Jésus. Faut-il soutenir que leur foi était certainement «jésuique» mais pas encore «chrétienne»? La question est bien difficile. Reconnaissaient-ils en Jésus le Messie? Certainement un prophète, le plus grand des prophètes devant apparaître avant la fin du monde. Y voyaient-ils la Parole définitive de Dieu? Oui, dans ses propres paroles, mais pas dans son destin. Des hérétiques, les premiers hérétiques? Qui en décidera?

En tant que mouvement, ces prophètes radicaux itinérants disparurent assez tôt de l'histoire du Christianisme. Mais pas sans laisser des traces durables: car, dans toute l'histoire du Christianisme, on peut déceler des individus, des groupes, des mouvements qui ont reproduit, à la marge des Eglises, les traits essentiels de leur message et de leur comportement.

Dès les origines donc, l'histoire de la foi se joue entre stabilité et rupture, dans une sorte de fluctuation qui poussera ses vagues jusqu'à nos jours.

La foi chrétienne est donc née à Pâques. Elle confesse le crucifié-ressuscité. Mais, m'objectera-t-on, ce n'est pas tout: elle confesse en son cœur Dieu le Père, le Fils et le Saint-Esprit, soit la Trinité. Nous observons cependant que le NT ne connaît pas ce dogme. Pas encore, faudrait-il écrire. Car la Trinité est le fruit d'une élaboration théologique, doctrinale des premiers siècles, qui trouve sa source dans la seule mention néotestamentaire du Père, du Fils et du Saint-Esprit tout à la fin de l'évangile de Matthieu: Mt 28, 19-20. Nous lisons là, dans la bouche du ressuscité, une formule liturgique baptismale de la première Eglise qui ne reflète pas encore une importante problématique doctrinale.

Dans les siècles qui suivront, par contre, de nombreux débats et des déchirements se cristalliseront autour du dogme de la Trinité. La plus sévère de ces controverses servira de motif théologique à la séparation entre les Eglises d'Orient et d'Occident. On l'appelle la querelle du filioque: il serait plus juste d'y reconnaître une polémique, à ce jour toujours pas éteinte, bien qu'atténuée par rapport à l'Antiquité et au Moyen-Age chrétiens. La formule latine cache que l'enjeu du débat est, de fait, la place reconnue à la troisième personne de la Trinité, au Saint-Esprit. Pour les Orientaux, le Saint-Esprit se juxtapose à égalité au Fils : ils reprochent aux Occidentaux de soumettre le Saint-Esprit en le faisant découler («procéder») du Père et du Fils. Querelle bien byzantine, serons-nous enclins à 
penser! Au fond, l'enjeu de la question est plus important qu'il n'y paraît au premier regard. Le souci des Eglises occidentales a toujours été de mettre des limites à ce qui pouvait être dit au nom de la foi. L'insistance sur le filioque allait dans ce sens. Elle voulait qu'on ne dise rien au nom du Saint-Esprit qui puisse contredire ce qui est donné à connaître par le Fils. Autrement dit: il ne faut pas permettre d'autre accès à Dieu que ce qu'il donne à connaître de lui-même dans sa Révélation historique en Jésus de Nazareth. Il n'y a pas d'expérience directe de Dieu, hors de la médiation de la Révélation en Jésus-Christ. Et de fait, dans le cours du temps, il s'est avéré que la divergence du Christianisme oriental par rapport à l'occidental s'est effectivement vérifiée aussi dans le rôle reconnu à Jésus-Christ. Généralement, le Christianisme occidental est plus centré christologiquement, l'oriental, l'Orthodoxie plus attachée aux expériences mystiques, aux extases provoquées, selon lui, par le contact direct de l'Esprit de Dieu. La théologie orthodoxe elle-même se montre plus liturgique que dogmatique, plus tournée vers l'expérience que vers la réflexion, à l'inverse des théologies catholiques-romaines et protestantes.

En nous arrêtant à quelques traces exprimant les stabilités et les fluctuations de la foi, nous avons dégagé l'idée qu'il doit bien y avoir dans la foi chrétienne un noyau stable, mais aussi beaucoup de fluctuations, sans que nous soyons à ce stade en mesure de préciser mieux notre première appréhension. A propos de la Trinité, nous pouvons reconnaître un facteur de stabilité dans le fait qu'autour de la même formule se sont développées et s'effectuent toujours des interprétations diverses. Mais nous devons aussitôt ajouter qu'aujourd'hui il se trouve des chrétiens qui s'affirment comme tels - certes une minorité - et qui refusent le dogme trinitaire et ne veulent adorer que Dieu le Père, reconnaissant à Jésus une fonction éminente mais non divine. Et nous avions noté qu'au départ de l'histoire de la foi chrétienne, des prophètes radicaux itinérants n'avaient accordé aucune valeur de salut à la passion et à la mort de Jésus. Si bien que notre question rebondit: où donc est la stabilité de la foi? Ou bien alors: existe-t-elle autrement que sous la forme de consensus dominants dans l'expression - qui cachent des conflits d'interprétation multiples - qui se dégagent peu à peu au cours du temps? Ou encore, plus gravement, la stabilité de la foi dépend-elle pour l'essentiel de ces consensus majoritaires qui excluent toute déviance, par leurs poids numériques, par leurs forces institutionnelles, par leurs capacités d'intimidation?

Afin d'augmenter notre perplexité, nous pouvons enrichir notre problématique d'un autre constat. Pouvons-nous exclure absolument que des expressions correctes, adéquates de la foi aient jamais caché des pratiques incorrectes, ainsi que l'inverse: qu'il n'y ait point eu d'expressions déviantes qui n'empêchaient pas des pratiques correctes? Une des critiques les plus élémentaires de la foi porte d'ailleurs précisément sur ce point, lorsqu'elle dit que, pour les chrétiens, et en particulier pour les gens pieux ou les ecclésiastiques, c'est: «faites comme je dis mais pas comme je fais!»

Les Eglises n'ont cessé de combattre les hérésies, parfois par les méthodes que l'on sait. Elles pouvaient bien avoir la saine doctrine, les armes par lesquelles elles ont voulu la défendre les ont parfois, trop souvent, disqualifiées. Si l'on pense au malheureux épisode cathare, on découvre cette tragique étrangeté, précisément. 
Les Cathares prônaient une doctrine dualiste, qu'ils voulaient d'inspiration chrétienne. Le Christianisme a toujours combattu le dualisme, avec raison: le dualisme religieux n'est pas compatible avec la Révélation de Dieu en JésusChrist. Et même l'évangile de Jean qui use de représentations dualistes en casse la métaphysique par son affirmation du prologue: «La Parole a été faite chair» (Jean $1,14)$. Mais les pratiques de vie cathares, pour autant qu'on les connaît, n'étaient pas si éloignées des recommandations évangéliques. La croisade et l'Inquisition ont constitué les réponses de l'Eglise officielle à l'hérésie cathare, au nom de la vraie doctrine (et de grands intérêts politiques, évidemment). Dans cette figure, la divergence entre les doctrines et les pratiques se montre abyssale. On pourrait retrouver dans chaque tradition de semblables inconséquences: dans son combat contre Avvakoum Petrov, l'Eglise Orthodoxe russe s'est aussi servie de moyens qui renient sa prétention à représenter le vrai Christianisme qu'elle professe. Et du côté protestant pensons, plus près encore de nous, à l'Afrique du Sud de l'apartheid et à l'Irlande.

Ce rappel peut nous rendre sensibles à une sorte de discontinuité à l'intérieur de la foi elle-même. D'une part il y a ses discours et, de l'autre, ce que nous avons appelé sa pratique, disons ce que la foi oriente et structure dans celui qu'elle habite. Où donc se trouve la priorité, s'il doit y en avoir une? Si nous pouvions répondre à cette question, nous nous trouverions vraisemblablement équipés pour repérer le noyau stable de la foi.

\section{FOI ET FOI :
L'HISTOIRE INTÉRIEURE ET EXTÉRIEURE DE LA FOI}

Une simple lecture des récits de guérisons de Jésus dans les évangiles nous fait constater qu'il y est question de foi, implicitement ou explicitement. L'exemple le plus éclatant est évidemment celui de l'épisode à la fin duquel Jésus déclare à la femme victime d'hémorragies chroniques: «Ma fille ta foi t'a sauvée; va en paix et sois guérie de ton mal.» (Marc 5, 34) Peut-être pourrons-nous apprendre ici de quelle foi il s'agit? Une relecture de l'ensemble de la péricope ${ }^{1}$ nous donne une réponse étrange: nous ne rencontrons aucune expression de foi de la part de la femme, ni avant, ni après sa guérison. Tout au plus, la femme se parle-t-elle à ellemême: «Si j'arrive au moins à toucher ses vêtements, je serai sauvée». Ce n'est

Marc 5, 24-34 : ${ }^{24}$ Jésus s'en alla avec lui; une foule nombreuse le suivait et l'écrasait. ${ }^{25}$ Une femme qui souffrait d' hémorragies depuis douze ans, ${ }^{26}$ - elle avait beaucoup souffert du fait de nombreux médecins et avait dépensé tout ce qu' elle possédait sans aucune amélioration; au contraire, son état avait plutôt empiré-, ${ }^{27}$ cette femme, donc, avait appris ce qu' on disait de Jésus. Elle vint parderrière dans la foule et toucha son vêtement. ${ }^{28}$ Elle se disait : Si j' arrive au moins à toucher ses vêtements, je serai sauvée. ${ }^{29}$ À l' instant sa perte de sang s'arrêta et elle ressentit en son corps qu' elle était guérie de son mal. ${ }^{30}$ Aussitôt Jésus s'aperçut qu' une force était sortie de lui. Il se retourna au milieu de la foule et il disait : "Qui a touché mes vêtements?» ${ }^{31}$ Ses disciples lui disaient : "Tu vois la foule qui te presse et tu demandes "Qui m' a touché?» ${ }^{32}$ Mais il regardait autour de lui pour voir celle qui avait fait cela ${ }^{33}$ Alors la femme, craintive et tremblante, sachant ce qui lui était arrivé, vint se jeter à ses pieds et lui dit toute la vérité. ${ }^{34}$ Mais il lui dit : "Ma fille, ta foi t'a sauvée; va en paix et sois guérie de ton mal.» 
pas une confession de foi mais l'expression d'un espoir insensé. Si bien que, pour Jésus qui, selon le narrateur, n'entend pas ce monologue, la foi de cette femme ne peut résider que dans son geste qu'il a perçu dans sa soudaine perte de «force». Cette femme tente ce geste qui pourrait presque être son dernier geste, son ultime démarche, la fin de ses tentatives, tant le texte a insisté auparavant sur la gravité de son mal et sur la vanité des multiples essais par lesquels elle a voulu se soigner. Et lorsque le texte souligne que «cette femme avait appris ce qu'on disait de Jésus» il ne faut pas imaginer autre chose que sa réputation de guérisseur. Evidemment que, dans les mentalités de l'époque, les pouvoirs thérapeutiques extraordinaires d'une personne qualifiaient ses rapports privilégiés avec la divinité (ou peut-être avec les forces mauvaises, cf. Marc 3, 20-30, polémique à propos de Beelzéboul). Mais rien de plus. La foi que Jésus détecte chez cette femme, c'est donc cet élan irraisonné qui l'a poussée vers lui, cette dernière démarche folle qui lui a fait croire qu'elle guérirait par Jésus, à travers Jésus ou même, plus précisément, par un contact avec son vêtement. On pourrait dire que l'auteur du récit de cette guérison accentue à plaisir les traits primitifs, naïfs, rudimentaires dont il est composé. Et la foi, ici, apparaît aussi dans son état le plus dépouillé, originel, élémentaire. Et si nous poussions l'enquête sur chaque récit de guérison de Jésus dans les évangiles, nous parviendrions dans toutes les occasions à des résultats analogues. Prenons, pour épreuve, un autre récit, mieux connu: celui de la guérison d'un enfant possédé, donc de cette catégorie spécifique des récits d'exorcismes (Marc 9, 14-27). L'enfant malade y apparaît presque comme un simple prétexte au dialogue de Jésus avec le père et à la guérison. Ici aussi le texte, par la voix du père et par la description de la crise, autant que par l'échec des disciples, se plaît à souligner l'envergure du mal. Tout se noue dans le dialogue de Jésus avec le père, autour de la foi. Jésus lui dit: «Tu peux! ... Tout est possible pour celui qui croit». Aussitôt le père de l'enfant s'écria: «Je crois! Viens au secours de mon manque de foi !» Cette dernière parole, dans une traduction légèrement différente («Viens au secours de mon incrédulité») a eu un énorme retentissement dans la piété et dans la littérature d'édification. Vraisemblablement parce qu'elle faisait résonner une corde profonde. Toujours la même corde: celle de la confiance ouverte, presque sans objet, celle de l'élémentaire confiance en la vie contre la mort, en l'humain contre ce qui le défigure.

Une puissante étude du genre littéraire des morceaux narratifs antiques que nous qualifions aujourd'hui de « récits de miracles » ${ }^{2}$ a montré que ceux-ci ont fait partie de la littérature populaire orale avant d'entrer dans des œuvres écrites, qu'ils manifestent une forme de protestation contre toutes les formes d'oppression par lesquelles l'existence des femmes et des hommes se trouvait réduite. Il faut s'imaginer que, dans les milieux particulièrement défavorisés, des conteurs s'adressaient à leurs contemporains en leur narrant des prodiges advenus à leurs semblables, et suscitaient de leur part des réactions d'enthousiasme ou de rejet. Ce faisant, ils réveillaient chez leurs auditeurs et en eux-mêmes à la fois un besoin de protestation contre le sort et une force irraisonnée de confiance malgré tout ce qui les accablait. Confiance dans le fait que la misère présente pouvait être surmontée.

2 Gerd Theissen, Urchristliche Wundergeschichten, Ein Beitrag zur formgeschichtlichen Erforschung der synoptischen Evangelien, Gütersloh, Gütersloher Verlagshaus Gerd Mohn, 1974. 
La structure même des récits de miracles opère ce dépassement. Ils partent tous d'une description plus ou moins accentuée de la misère et de la détresse, font apparaître les éléments susceptibles d'autoriser le dépassement de cette situation puis donnent des traits manifestant la restitution d'une situation normale, avant de conclure par l'attestation de témoins du passage de l'un à l'autre état. Et le récit lui-même veut opérer une telle mutation chez ceux qui l'entendent et le reçoivent. Et, du côté des victimes, l'élément qui va permettre la transformation de la situation est toujours la foi. La foi sous la forme de demande de secours, d'expression de confiance envers et contre tout ou de simple prière, parfois provoquée par le faiseur de miracle lui-même. Dans les récits de miracles chrétiens, c'est évidemment Jésus qui joue ce rôle.

Nous pouvons donc dire que les récits de guérison du Nouveau Testament rapportés à Jésus dessinent les traits les plus élémentaires de ce qu'il convient d'appeler «la foi ». Théologiquement, à ce stade, on peut la dire indéterminée. Par rapport à la détresse, au désespoir, la foi veut croire contre toute apparence, que la vie humaine est encore possible. Et elle en appelle à celui qui porte une réputation de thérapeute, au fond de réparateur de la condition humaine.

La foi transmise par les récits de miracles exprime deux choses: une expérience de l'oppression manifeste dans la réduction de la vie humaine à ses plus basses limites; une expérience du retournement encore possible. Que Dieu, disons la Puissance Supérieure ou même Suprême, soit à l'origine d'un tel retournement est sous-entendu comme une évidence.

Pour cette tradition chrétienne élémentaire (celle des «récits de miracles»), Jésus est celui par qui Dieu lui-même opère le retournement. Ceux qui la rapportent en ont bénéficié évidemment de manière directe ou indirecte, par expérience.

Mais, contrairement à ce qui se produit pour d'autres thérapeutes antiques ${ }^{3}$, la chose, évidemment, se complique en Christianisme du fait de la mort de Jésus et de la signification théologique qu'on lui attribue: là, dans un premier temps, les adhérents de Jésus ont dû faire l'amère expérience d'une totale et calamiteuse absence de miracle. Et l'on trouve l'écho de cette désillusion dans les récits de la Passion eux-mêmes, par exemple dans cette moquerie des passants: «Hé! Toi qui détruis le Sanctuaire et le rebâtis en trois jours, sauve-toi toi-même en descendant de la croix.» (Marc 15, 30) Dans sa spontanéité, la foi s'est heurtée dès l'origine à cet obstacle. Et l'expérience de la Résurrection - l'expérience des visions, des apparitions dont les principaux adhérents désillusionnés ont bénéficié, qui leur présentaient le déchu comme le victorieux - a opéré un nouveau retournement, au sens d'un passage de l'oppression à la libération, du désespoir à la foi.

A grands traits et sur un modèle abstrait de ce qui s'est vraisemblablement passé pour la première conscience chrétienne, nous venons d'esquisser une sorte d'itinéraire entre l'expérience de la foi élémentaire illustrée dans les récits de miracles et celle du choc d'un réel qui la dément. Exprimé en mots, cet itinéraire témoigne d'une dialectique de l'expérience et de la réflexion théologique, disons de sa ressaisie. Et nous trouvons dans cette dialectique les indices de la discontinuité que j'exposais en fin de première partie. Cette dialectique et la discontinuité dont elle rend compte sont essentielles à la constitution de la foi.

Apollonius de Tyane, par exemple, cf. Philostrate. 
Depuis quelques siècles, les théologiens opèrent volontiers une distinction dans la foi qui rejoint ce que nous venons d'établir: la fides qua creditur n'est pas la fides quae creditur, la foi par laquelle on croit ne doit pas être confondue avec ce qu'on croit. Je veux penser cette distinction essentielle et éclairante. La foi par laquelle on croit (fides qua creditur) rejoint cette confiance irraisonnée, ouverte, tout à l'origine théologiquement encore indéterminée, qui caractérise la demande de guérison dans les récits de miracles. La tradition protestante a toujours insisté sur le postulat que cette foi-là ne pouvait qu'être reçue, jamais conquise, qu'elle ne résultait ni d'un mérite ni d'un effort: qu'elle apparaissait comme le premier don de Dieu, le vrai don du ciel, pour reprendre la formulation de notre question de départ. Mais elle n'apparaît à l'évidence comme telle que rétrospectivement, dans la relecture de la propre histoire du croyant. En elle-même, par elle-même, dans sa spontanéité et son immédiateté, elle ne constitue qu'un épisode incapable de se comprendre. Elle doit encore s'interpréter elle-même dans la rencontre de sa référence et dans le choc du réel, ici du temps et de l'histoire. Et cette interprétation est évidemment une tâche continue. Lorsque, dans le récit que nous avons lu, Jésus dit à la femme: «Ma fille, ta foi t'a sauvée; va en paix et sois guérie de ton mal », il la renvoie à elle-même, il lui ouvre la possibilité de revenir sur cet élan qui l'a poussée malgré tous les obstacles - les consultations antérieures catastrophiques, l'aggravation de son mal et, dans l'instant, la foule qui la sépare de Jésus - à chercher le contact. La foi irraisonnée est renvoyée à son propre approfondissement. L'une appelle l'autre comme son complément nécessaire, la fides qua implique une fides quae; la première sans la seconde se réduit à un incident sans suite, mais la seconde sans la première est vaine et sans fondement. Car la première est l'essentielle, hors de toute maîtrise.

\section{CONCLUSION}

Je vous propose de penser que la seule stabilité de la foi chrétienne réside précisément dans cette tension entre la foi en tant que don et la foi en tant qu'expression, dans cette dialectique de l'expérience originaire de la confiance envers et contre tout et de la réflexion qui la rapporte à Dieu, à travers l'aspérité de la Révélation paradoxale du crucifié. Etant bien entendu que le processus s'effectue dans la rencontre du réel, dans le cours du temps, dans des fluctuations. Ainsi s'expliquent les continuités et les ruptures, les stabilités et les fluctuations que nous avions notées en ouverture. Derrière tout cela se développent des expériences et des interprétations qui s'expriment, par exemple, dans des confessions de foi, des aveux individuels, et qui alimentent aussi des conflits. Car, dans son retour sur elle-même, la foi élémentaire menace toujours de dévier, de dériver, de se faire, par exemple, hallucination ou bien illumination ou doctrinaire fanatique. Elle a besoin d'une discipline qui la structure, qui la développe dans le cours du temps, qui la tienne authentique dans les limites du réel. Elle a besoin de stabilité, mais d'une stabilisation dynamique autant que possible. Elle la trouve dans sa tâche d'autointerprétation par l'interprétation des témoignages extérieurs, d'un récit de miracle, par exemple. Et il va de soi que, dans son développement au cours du temps, le devenir croyant ne se limite pas à des expériences de confiance ouverte dans la vie et à leurs ressaisies dans une réflexion rétrospective. En observant les récits de 
guérison, nous avions déjà noté la dimension de protestation qui accompagne l'expérience de la confiance. Elle indique que la foi proteste parfois, souvent contre sa propre absence, en même temps que contre la fermeture du monde sur lui-même et contre la réduction de la vie humaine aux frontières de l'absurde, pour des raisons de santé mais aussi pour des motifs économiques, sociaux et politiques. Ainsi ce devenir doit-il être conçu comme un mixte de certitudes et de doutes, d'expériences de résonance et d'expériences d'absurdité, en même temps que comme un courage incessant de retour à soi et de révision continue.

Par conséquent, dans sa rencontre des textes anciens, d'une autre époque, la foi s'avoue qu'elle doit en reprendre l'interprétation dans les circonstances qui la situent elle-même. Les récits de miracles du NT sont très dépendants de leur époque dans leur genre littéraire, dans leurs représentations, dans leur contexte de communication. Mais, dans leur anachronisme même, ils expriment quelque chose d'essentiel qu'on peut retrouver aujourd'hui. Je pense précisément à la représentation de la foi comme simple besoin d'ouverture à la vie humaine possible et à un monde habitable, envers et contre tout. Ce besoin est archaïque, non pas tant dans un sens de pur chronologie, mais existentiel. Il habite l'humanité, comme le montrent toutes les protestations qui s'élèvent contre les conditions faites à l'existence des humains. C'est donc à un tel besoin de confiance envers et contre tout, à un tel appel à une promesse qui rendrait sensée l'existence de chacun, que prétend répondre la foi chrétienne.

Pour des raisons évidentes, les récits de miracles chrétiens primitifs insistent toujours sur la réduction de la vie humaine à ses limites inférieures. Sans qu'on puisse prétendre que ces aliénations aient été aujourd'hui toutes réduites, il nous faut noter que l'actualité technique et scientifique nous ajoute des problèmes inverses qui se situent vers les limites supérieures. Alors que les guérisons mises en récit dans le NT ne nous laissent jamais penser que les personnes rétablies - ou même ressuscitées - puissent vivre autre chose qu'une vie humaine normale, c'està-dire limitée, certaines recherches contemporaines convergent pour orienter nos attentions et, si possible, nos désirs vers l'utopie d'une vie humaine qui aurait surpassé certaines de nos limites décisives, outrepassé la finitude. Mais on ne peut pas prétendre que le NT ait été totalement insensible à ce type d'aliénation: d'abord la tradition religieuse judéo-chrétienne a constamment dénoncé la volonté de maîtrise divine des humains; ensuite, plus concrètement, le récit d'exorcisme le plus brut, le plus pur aussi du NT, dénonce précisément cette utopie. Le démoniaque de Gérasa (Marc 5, 1-20) n'illustre-t-il pas, dans les détails de sa folie, cette tendance à vouloir aller au-delà de toute limite qui caractérise la condition humaine? Ce texte est porteur d'une protestation dont les aspects politique (contre l'empire romain), économique (contre le calcul marchand de la valeur des porcs payée pour le rétablissement d'un humain) et social (contre l'angoisse du dérangement causé par la guérison d'un humain) pourraient bien rejoindre nos indignations. Le miracle consiste aujourd'hui, comme hier, dans l'acceptation de l'existence humaine mortelle. Et ce miracle ne peut advenir qu'à travers la confiance élémentaire, ouverte à la vie envers et contre tout. Cette confiance, la foi chrétienne la lie à Jésus de Nazareth reconnu comme Christ dans sa mort même.

Les résultats auxquels nous aboutissons sont, pour beaucoup, spécifiques à une saisie chrétienne de la foi. A l'évidence, on pourrait trouver des parentés dans 
la foi juive. Beaucoup moins dans d'autres traditions religieuses, certainement. La foi chrétienne présente ou devrait présenter cette particularité que ce qui fait en elle l'essentiel est hors de la maîtrise des hommes et même du croyant, et que tout le reste, en particulier ses expressions, est révisable, soumis aux aléas. La foi chrétienne porte en elle sa propre dimension critique, son propre antidote au fanatisme. Il lui reste pratiquement à l'exercer, dans le temps, comme un élément essentiel de sa stabilité. Elle ne l'a pas toujours fait, rompant ainsi la dialectique, croyant vaincre la tension, et elle ne semble pas toujours vouloir le faire: c'est que sa stabilité en tension est difficile à maintenir existentiellement. Le domaine religieux est toujours tenté de confondre la foi avec des expressions de certitudes, de formulations ou de dogmes absolus.

Faculté de théologie

Université de Neuchâtel

pierre-luigi.dubied@unine.ch 